\title{
Effect Of Fault Resistance On The Performance Of Mho Relays
}

\author{
Noha Abed Al-bary Al-jawady \\ Department of Technical Power Engineering - Technical \\ College/Mosul
}

\begin{abstract}
For distance protection to perform its function perfectly without errors, it should be characterized with ideal characteristics that is to operate within forward faults those are included by protection zone, and to exclude the outer faults.

In order to get an ideal tripping area for distance relays, all the factors and limitations imposed by power system on the measuring accuracy of distance relays must be identified.

This paper includes a study of the fault resistance effect on the performance of distance protection when using mho relays.

This study is done by steady state analysis of fault circuit to find the current \& voltage at the relay location for different value of fault resistance for Line to ground faults and double line to ground faults.
\end{abstract}

Keywords: fault resistance, Protective relays, Mho relays.

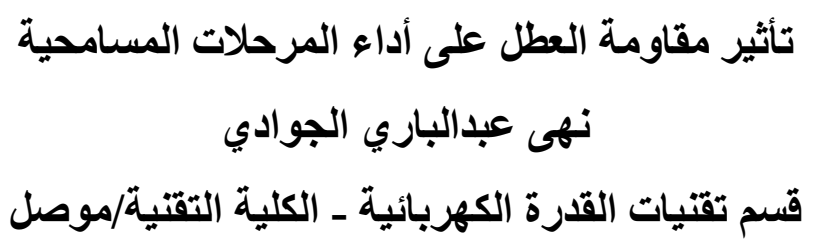

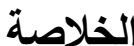

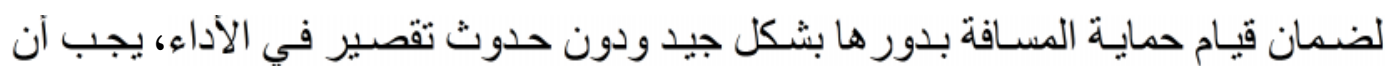

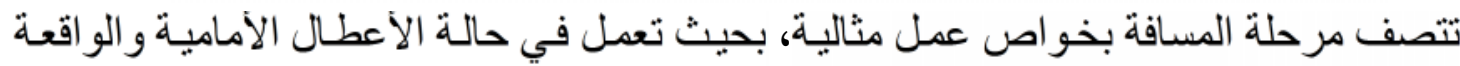


ضمن القطاع المحمي من قبل المرحلة وتستثني الأعطال التي تقع خارج القطاع المحمي من قبل

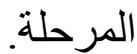

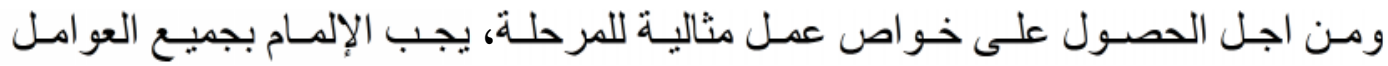
و المحددات المفروضة من قبل منظومة القدرة والتي تؤثر على عمل المرحلة.

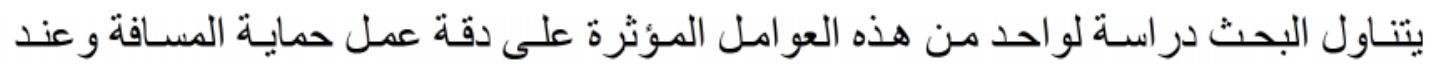

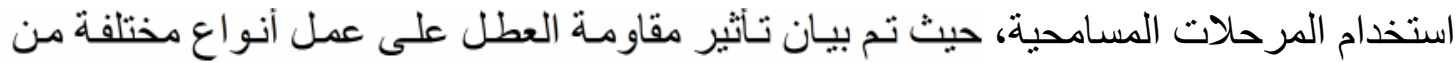

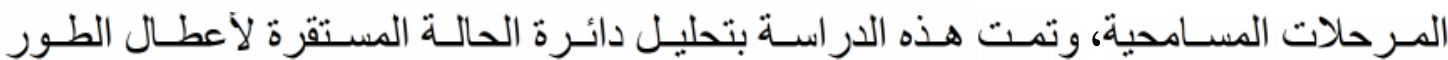
و الطورين مع الأرض و عند قيم مختلفة لمقاومة العطل.

\section{Types DfcSingyld Phalse2106ho Relays}

Accepted 16 July 2007

The operating characteristic of mho elements are derived either by amplitude or phase comparator of two vectors derived from the voltage and current signals of the protected line. But the most available distance relays derive a mho characteristic from two input phase comparators, which operate when the phase angle between two vectors $S_{1}$ and $S_{2}$ lies between $\Pi / 2 \& 3 \Pi / 2$. Theses two vectors are:-

$$
\mathrm{S}_{1}=\mathrm{K}_{1} \mathrm{~V}_{\Phi}-\mathrm{I}_{\Phi} \mathrm{Z}_{\mathrm{R}}
$$

$$
\mathrm{S}_{2}=\mathrm{K}_{2} \mathrm{~V}_{\Phi}-\mathrm{K}_{3}
$$$$
\mathrm{I}_{\Phi} \mathrm{Z}_{\mathrm{R}}+\mathrm{K}_{4} \mathrm{~V}_{\mathrm{p}}
$$

Where :-

$Z_{R}$ is the relay impedance setting.

$\mathrm{V}_{\Phi}, \mathrm{I}_{\Phi}$ are the voltage \& current at relay location .

$\mathrm{K}_{1}, \mathrm{~K}_{2}, \mathrm{~K}_{3} \& \mathrm{~K}_{4}$ are real or complex constants.

$\mathrm{V}_{\mathrm{p}}$ is a polarizing voltage.

Three typical mho elements are considered which are currently used in electronic distance relays and which exemplify the various form of 
polarization. A non polarized mho element is studied for comparison purpose, and these types are classified as reference [ 1 ]. These types are:1)Non polarized. Which is characterized by :

$\mathrm{K}_{1}=\mathrm{K}_{2}=1, \mathrm{~K}_{3}=\mathrm{K}_{4}=0$

2)Lightly cross polarized, Which is characterized by :

$\mathrm{K}_{1}=\mathrm{K}_{2}=1, \mathrm{~K}_{3}=0.001$ for offset in the positive direction, $\mathrm{K}_{4}=0.09$ to provide cross

polarization .

3)Moderately cross polarized, Which is characterized by :

$\mathrm{K}_{1}=\mathrm{K}_{2}=1, \mathrm{~K}_{3}=0, \mathrm{~K}_{4}=-0.98<60^{0}$ for $\Phi-\mathrm{G}$ element, $\mathrm{K}_{4}=-0.33<85$ for $\Phi-\Phi$ elements

4)Strongly cross polarized plus memory polarized Which is characterized by :

$\mathrm{K}_{1}=1, \mathrm{~K}_{2}=\mathrm{K}_{3}=0, \mathrm{~K}_{4}=1$.

Where $V_{p}$ is selected such that is composed of $80 \%$ of positive sequence fault

voltage $\& 20 \%$ pre-fault voltage at relay location.

\section{The Response Of Mho Relay}

The response of mho relay can be found by using of one of these methods

1-Loop impedance method.

2-Line impedance method.

3-Apparent impedance method.

The simplest $\&$ the best method for finding the response of mho relay is the third one (apparent impedance method), it requires the calculation of an apparent impedance only (impedance measuring by the relay), as given in appendix (A) with characteristic is a circle passing through the origin, reach point, invariant with the fault type \& system parameter [2 ].

In order to find the voltage \& current at relay location before $\&$ after fault occurrence SPICE program has been used for the analysis of steady-state fault circuits.

To achieve the phase voltages \& phase currents from sequences voltages \& sequences currents the following equation are used.

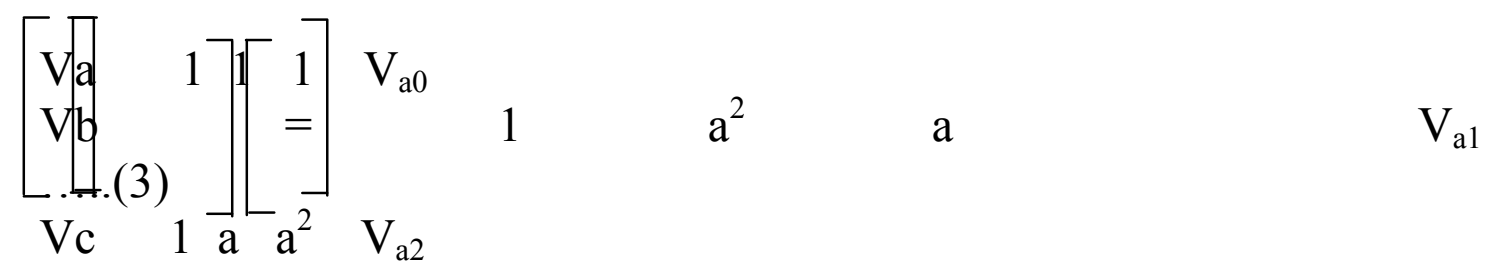


By using equation $\left(A_{6}\right)$ the apparent impedance for the types of mho relays can be obtained.

\section{Effect Of Fault Resistance}

In the cases of lines short circuits, the fault resistance are small $\&$ do not exceed a few ohms. However, it may become much higher during ground faults owing to the tower footing $\&$ arc resistance.

In order to show the effect of polarizing and fault resistance on the response of mho elements, the response of the four typical mho elements has been found which exemplify the various forms of polarizing at location ( 1 ) as shown in fig.(1), which represent transmission line between two stations at rated voltage of $(15 \mathrm{kV})$, when two lines to ground fault \& line to ground fault occurs at different locations of T.L.[3]. Different fault location are examined $\left(\mathrm{F}_{1}, \mathrm{~F}_{2}, \mathrm{~F}_{3}\right)$ such that:-

$\mathrm{F}_{1}$ : fault at (1/4) of T.L

$F_{2}$ : fault at $(1 / 2)$ of T.L.

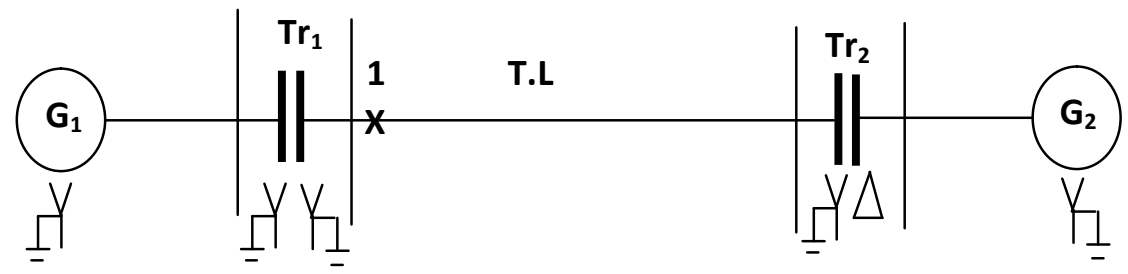

Fig.(1) single line diagram of power system

The MVA rating, voltage rating, and sequence impedances of the element of fig.(1) are listed in table (1) in appendix (C).

\section{1-Single Line To Ground Fault (LG).}

In general the single line to ground fault on a transmission system occurs when one conductor falls to ground or contacts the neutral wire.

Figure(2) (in appendix (D)) shows the interconnection of the resulting sequence networks when LG fault occur.

Table (2) (in appendix (C))shows an apparent impedance for four types of mho relay (which are calculated by using equation $\left(\mathrm{A}_{6}\right)$ ) in perunit to the relay impeding setting $\left(Z_{R}\right)$ (when $Z_{R}$ is $80 \%$ of T.L and $120 \%$ of T.L) as abase impedance, for different values of fault resistance $R_{F}$ 
which is suggest resistance for case studying, when L-G occurs at different location on T.L,

Where $\mathrm{Za}_{1}=$ apparent impedance for non polarized type.

$\mathrm{Za}_{2}=$ apparent impedance for Lightly cross polarized type.

$\mathrm{Za}_{3}=$ apparent impedance for Moderately cross polarized.

$\mathrm{Za}_{4}=$ apparent impedance for Strongly cross polarized plus memory polarized.

The response of mho relays at location (1) when L-G fault occur at positions $\left(\mathrm{F}_{1}, \mathrm{~F}_{2} \& \mathrm{~F}_{3}\right)$ is shown in figures ( $4 \mathrm{a}, 4 \mathrm{~b}, \& 4 \mathrm{c}$ respectively).

It is shown in fig.(4a), that $Z_{a 1}, Z_{a 2} \& Z_{a 3}$ become outside the relay characteristic zone when $\mathrm{R}_{\mathrm{F}}$ equal to $(10 \Omega)$, while $Z_{\mathrm{a} 4}$ remain inside the characteristic zone and becomes outside when $\mathrm{R}_{\mathrm{F}}$ equal to $(20 \Omega)$.

In fig $(4 \mathrm{~b}$ and $4 \mathrm{c}) \mathrm{Z}_{\mathrm{a} 1}, \mathrm{Z}_{\mathrm{a} 2} \& \mathrm{Z}_{\mathrm{a} 3}$ become outside the relay characteristic when $R_{F}$ equal to $(10 \Omega)$, while $Z_{a 4}$ remain inside the characteristic and it $s$ become outside when $R_{F}$ equal to $(15 \Omega)$

\section{2-Double Line To Ground Fault.}

In general, the double Line to ground (DLG) fault on a transmission system occurs when two conductors fall and are connected through ground or when two conductors contact the neutral of a three phase grounded system.

Figure(3)(in appendix (D)) shows the interconnection of the resulting sequence networks when DL-G fault occur.

Table (3) ( in appendix $(C)$ shows an apparent impedance for four types of mho relay in per-unit to the relay impeding setting $\left(Z_{R}\right)$ (as abase impedance)for different values of fault resistance when DL-G occurs at different location on T.L .

The response of mho relays at location (1) when DL-G fault occur at positions $\left(\mathrm{F}_{1}, \mathrm{~F}_{2} \& \mathrm{~F}_{3}\right)$ is shown in figures $(5 \mathrm{a}, 5 \mathrm{~b}, \& 5 \mathrm{c}$ respectively).

It is see that in fig.(5a), $Z_{a 1}, Z_{a 2}, Z_{a 3} \& Z_{a 4}$ remain inside the relay characteristic when increase $R_{F}$ to $(20 \Omega)$ and in fig $(4 b$ and $4 c) Z_{a 1} \& Z_{a 2}$ become outside the relay characteristic when $R_{F}$ equal to $(20 \Omega)$, while $Z_{a 3}$ $\& \mathrm{Z}$ a4 remain inside the characteristic 


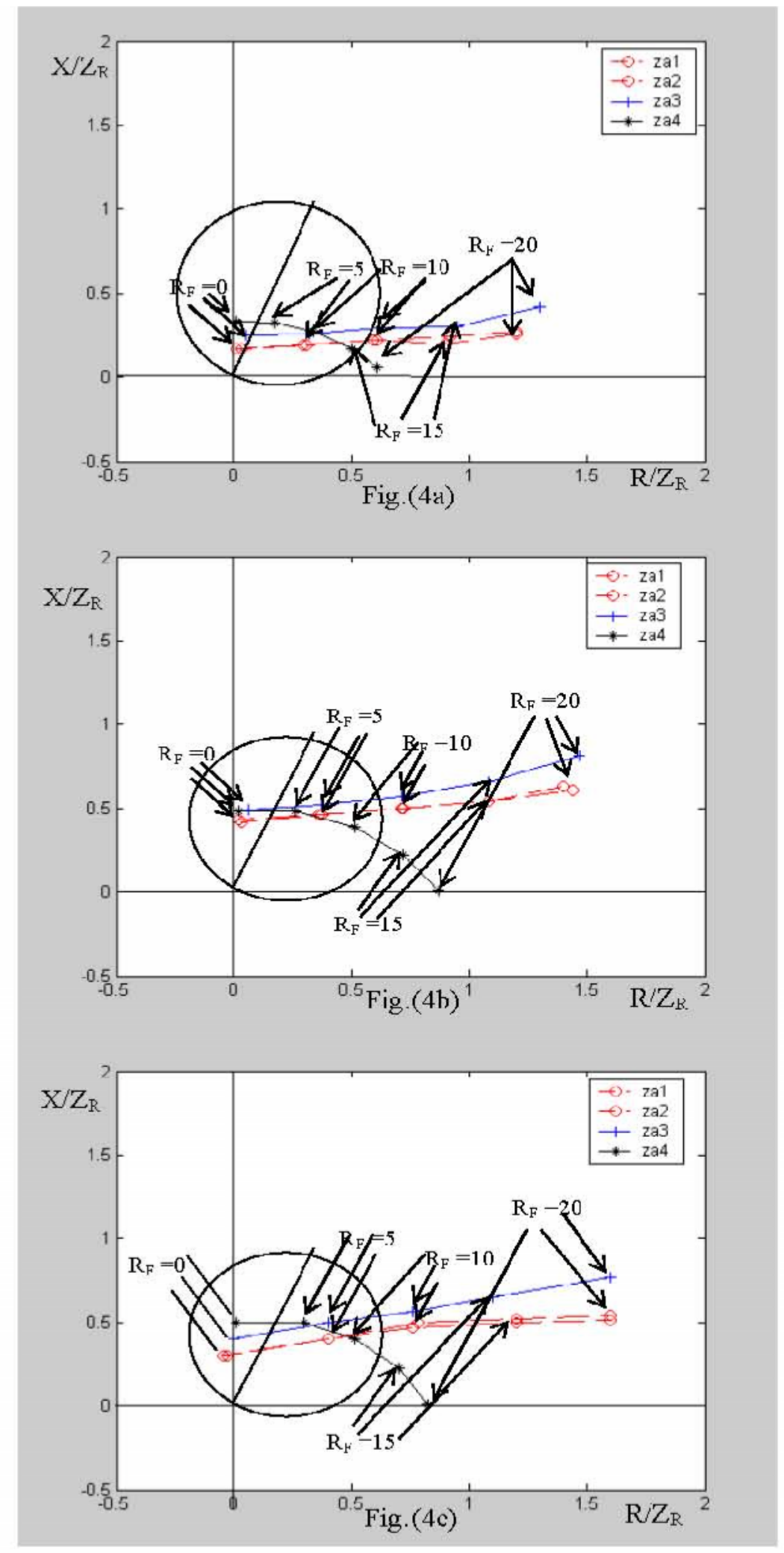

Fig.(4)'The response of mho elements when LG fault occurs for different value of fault resistance (a) Fault at $\Gamma_{1}$ (b) $\Gamma$ ault at $\Gamma_{2}$ (c) $\Gamma$ ault at $\Gamma_{3}$. 


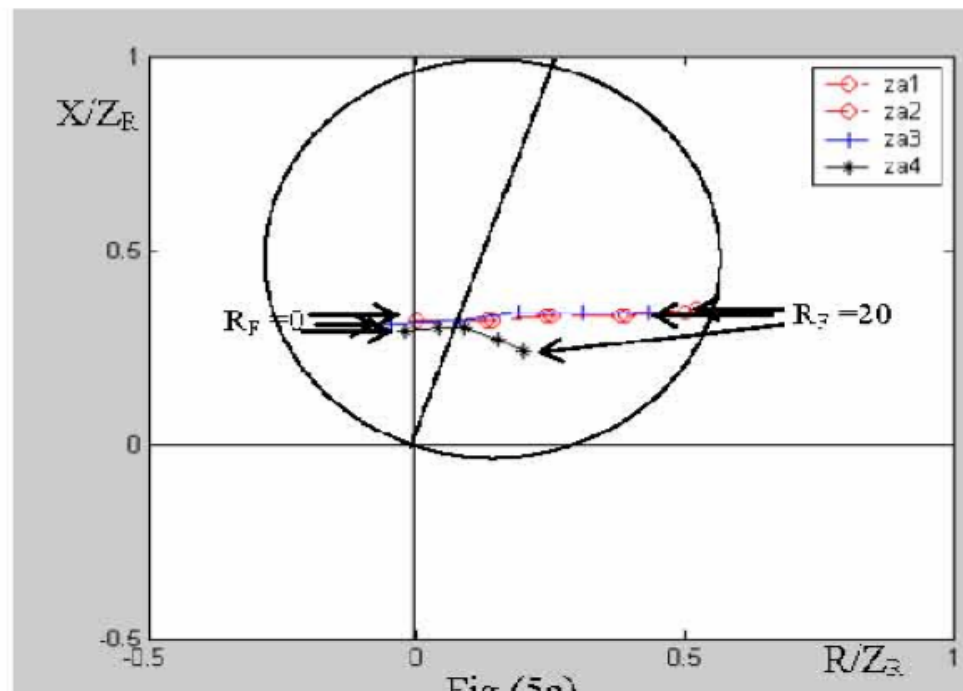

Fig.(5a)
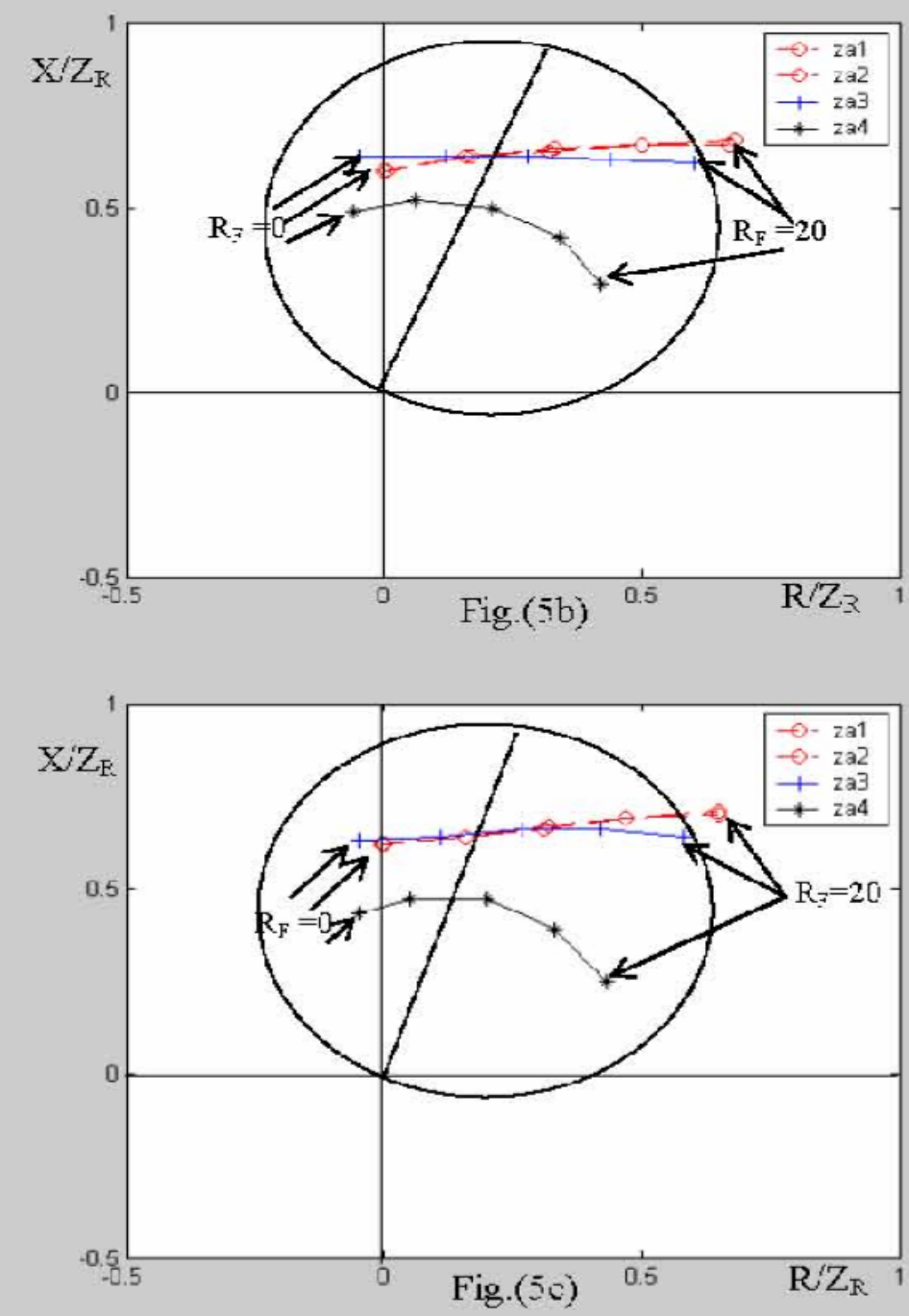

Fig.(5) The response of mho elements when $D L G$ fault occurs for different value of resistance (a)Fault at $F$ : (b)Fault at $F_{2}$ (c) Fault at $F_{3}$.

\section{Conclusion}


The results on the performance of mho elements after analysis for steady state fault circuits (LG-fault \& DLG-fault) for different value of fault resistance $\left(\mathrm{R}_{\mathrm{F}}\right)$ indicate that:-

1-As a fault resistance has been increased, the apparent impedance of mho elements moves out of the characteristic and this, means that the relay will fail to operate (under reach)

2-The effect of $\left(R_{F}\right)$ on the directional of mho element in case of LGfault was more than in case of $2 \mathrm{LG}$-fault.

3- The effect of $\left(\mathrm{R}_{\mathrm{F}}\right)$ on the directional of mho element was decreased when the fault is more nearer to the relay location.

4-Apparent impedance measured by mho relay became $\left(\mathrm{Z}_{\mathrm{F}}+\mathrm{R}_{\mathrm{F}}\right)$ instead of $Z$ where $Z_{F}$ is the impedance between relay \& fault location. From these results it was evident that, at a certain value of fault resistance, some of mho elements became non directional (apparent impedance becomes outside of the relay characteristic) while other types was still directional (apparent impedance still inside the relay characteristic). On the other hand, the response of mho element depending on the type of polarization.

\section{Reference}

1-R.J.Marttila, "Directional Characteristic of Distance Relay Mho Elements" Part I.A new method of analysis,Part II.Results IEEE Trans. On power Apparatus and System,Vol.PAS-100,No.1, January 1981.

2- Noha Abed Al-bary Al-Jawady, "Effect of loading on the performance of mho relays with two phases fault" M.Sc. Thesis, University of Mosul, 1998.

3-Turan Gonen, "Modern Power System Analysis" John Wiley \& Sons, INC. 1988

\section{Appendix (A)}

\section{Calculation of apparent impedance}

An apparent impedance method of representing mho element response can be derived from the fact that the mho characteristic always passes through the origin and the reach point if $\mathrm{k}_{3} \& \mathrm{k}_{4}$ are zero. Thus an alternate set of phase comparison vectors, such as: 


$$
\begin{aligned}
& \bar{S}_{1}=\bar{V}_{\phi}-\bar{I}_{\phi} Z_{R} \\
& \bar{S}_{2}=\bar{V}_{\phi}
\end{aligned}
$$

Would define a mho characteristic on the $\bar{Z}_{\phi}=\mathrm{R}+\mathrm{jX}$ plane having these features. This characteristic would define the range of impedance ( $\left.\bar{Z}_{\phi}\right)$ for which the mho element operates. The general phase comparison vectors, $\mathrm{S}_{1} \& \mathrm{~S}_{2}$ can be transformed into vectors $\bar{S}_{1} \& \bar{S}_{2}$ by defining.

$$
\begin{aligned}
& \bar{V}_{\phi}=K_{2} V_{\phi}+K_{3} I_{\phi} Z_{R}+K_{4} V_{P} \\
& \bar{I}_{\phi}=I_{\phi}\left(1+K_{3}\right)+\frac{K_{4} V_{P}}{Z_{R}}+\frac{K_{2}-K_{1}}{Z_{R}} V_{\phi}
\end{aligned}
$$

With this transformation, all mho elements willhave a fixed characteristic represented by a circle passing through the origin and the reach point $Z_{R}$ on the $\bar{Z}_{\phi}$ plane, $\bar{Z}_{\phi}$ is referred to as an apparent impedance and is defined as :

$$
\bar{Z}_{\phi}=\frac{\bar{V}_{\phi}}{\bar{I}_{\phi}}=\frac{K_{2} V_{\phi}+K_{3} I_{\phi} Z_{R}+K_{4} V_{P}}{I_{\phi}\left(1+K_{3}\right)+\frac{K_{4} V_{P}+\left(K_{2}-K_{1}\right) V_{\phi}}{Z_{R}}}
$$

And from this equation we see that:

$$
\bar{Z}_{\phi}=Z_{R} \frac{S_{2}}{S_{2}-S_{1}}
$$


To determine the response of any mho element to any fault type, this apparent impedance is calculated and compared with the fixed circular characteristic on the $\bar{Z}_{\phi}$ plane.

\section{Appendix (B)}

\section{Operating equations for mho element types}

\section{Mho element No.1}

Operating equations:-

$$
\begin{aligned}
& \mathrm{S}_{1}=\mathrm{V}_{\Phi}-\mathrm{I}_{\Phi} \mathrm{Z}_{\mathrm{C}} \\
& \mathrm{S}_{2}=\mathrm{V}_{\Phi}
\end{aligned}
$$

Where $Z_{C}$ is reach setting impedance.

The variable for the various element types are listed below.

\begin{tabular}{|l|l|l|}
\hline Element type & $\mathrm{I}_{\Phi}$ & $\mathrm{V}_{\Phi}$ \\
\hline A-G & $\mathrm{I}_{\mathrm{A}}+\mathrm{k}_{\mathrm{o}} \mathrm{I}_{\mathrm{N}}$ & $\mathrm{V}_{\mathrm{A}}$ \\
\hline $\mathrm{B}-\mathrm{G}$ & $\mathrm{I}_{\mathrm{B}}+\mathrm{k}_{\mathrm{o}} \mathrm{I}_{\mathrm{N}}$ & $\mathrm{V}_{\mathrm{B}}$ \\
\hline $\mathrm{C}-\mathrm{G}$ & $\mathrm{I}_{\mathrm{C}}+\mathrm{k}_{\mathrm{o}} \mathrm{I}_{\mathrm{N}}$ & $\mathrm{V}_{\mathrm{C}}$ \\
\hline A-B & $\mathrm{I}_{\mathrm{AB}}$ & $\mathrm{V}_{\mathrm{AB}}$ \\
\hline B-C & $\mathrm{I}_{\mathrm{BC}}$ & $\mathrm{V}_{\mathrm{BC}}$ \\
\hline C-A & $\mathrm{I}_{\mathrm{CA}}$ & $\mathrm{V}_{\mathrm{CA}}$ \\
\hline
\end{tabular}

Where $\mathrm{k}_{\mathrm{o}}=1 / 3\left(\mathrm{Z}_{0} / \mathrm{Z}_{1}-1\right)=$ neutral compensation.

\section{Mho element No.2}


Operating equations:-

$\mathrm{S}_{1}=\mathrm{V}_{\Phi^{-}} \mathrm{I}_{\Phi} \mathrm{Z}_{\mathrm{C}}$

$\mathrm{S}_{2}=\mathbf{V}_{\Phi}+\mathrm{k}_{3} \mathrm{I}_{\Phi} \mathrm{Z}_{\mathrm{C}}+\mathrm{k}_{4} \mathrm{~V}_{\mathrm{p}}$

\begin{tabular}{|l|l|l|l|l|l|}
\hline Element type & $\mathrm{I}_{\Phi}$ & $\mathrm{V}_{\Phi}$ & $\mathrm{V}_{\mathrm{p}}$ & $\mathrm{k}_{3}$ & $\mathrm{k}_{4}$ \\
\hline A-G & $\mathrm{I}_{\mathrm{A}}+\mathrm{k}_{\mathrm{o}} \mathrm{I}_{\mathrm{N}}$ & $\mathrm{V}_{\mathrm{A}}$ & $\mathrm{V}_{\mathrm{BC}}$ & -0.001 & 0.02 \\
\hline B-G & $\mathrm{I}_{\mathrm{B}}+\mathrm{k}_{\mathrm{o}} \mathrm{I}_{\mathrm{N}}$ & $\mathrm{V}_{\mathrm{B}}$ & $\mathrm{V}_{\mathrm{CA}}$ & -0.001 & 0.02 \\
\hline $\mathrm{C}-\mathrm{G}$ & $\mathrm{I}_{\mathrm{C}}+\mathrm{k}_{\mathrm{o}} \mathrm{I}_{\mathrm{N}}$ & $\mathrm{V}_{\mathrm{C}}$ & $\mathrm{V}_{\mathrm{AB}}$ & -0.001 & 0.02 \\
\hline $\mathrm{A}-\mathrm{B}$ & $\mathrm{I}_{\mathrm{AB}}$ & $\mathrm{V}_{\mathrm{AB}}$ & $\mathrm{V}_{\mathrm{CA}}$ & -0.001 & -0.02 \\
\hline $\mathrm{C}-\mathrm{A}$ & $\mathrm{I}_{\mathrm{BC}}$ & $\mathrm{V}_{\mathrm{BC}}$ & $\mathrm{V}_{\mathrm{AB}}$ & -0.001 & -0.02 \\
\hline & $\mathrm{I}_{\mathrm{CA}}$ & $\mathrm{V}_{\mathrm{CA}}$ & $\mathrm{V}_{\mathrm{BC}}$ & -0.001 & -0.02 \\
\hline
\end{tabular}

\section{Mho element No.3}

Operating equations:-

$$
\begin{aligned}
& \mathrm{S}_{1}=\mathrm{V}_{\Phi}-\mathrm{I}_{\Phi} \mathrm{Z}_{\mathrm{C}} \\
& \mathrm{S}_{2}=\mathrm{V}_{\Phi}+\mathrm{k}_{4} \mathrm{~V}_{\mathrm{p}}
\end{aligned}
$$

\begin{tabular}{|l|c|c|c|c|}
\hline \multicolumn{1}{|c|}{$\begin{array}{r}\text { Element } \\
\text { type }\end{array}$} & $\mathrm{I}_{\Phi}$ & $\mathrm{V}_{\Phi}$ & $\mathrm{V}_{\mathrm{P}}$ & $\mathrm{k}_{4}$ \\
\hline $\mathrm{A}-\mathrm{G}$ & $\mathrm{I}_{\mathrm{A}}+\mathrm{k}_{\mathrm{o}} \mathrm{I}_{\mathrm{N}}$ & $\mathrm{V}_{\mathrm{A}}$ & $\mathrm{V}_{\mathrm{C}}$ & $-0.28<60$ \\
\hline $\mathrm{B}-\mathrm{G}$ & $\mathrm{I}_{\mathrm{B}}+\mathrm{k}_{\mathrm{o}} \mathrm{I}_{\mathrm{N}}$ & $\mathrm{V}_{\mathrm{B}}$ & $\mathrm{V}_{\mathrm{A}}$ & $-0.28<60$ \\
\hline $\mathrm{C}-\mathrm{G}$ & $\mathrm{I}_{\mathrm{C}}+\mathrm{k}_{\mathrm{o}} \mathrm{I}_{\mathrm{N}}$ & $\mathrm{V}_{\mathrm{C}}$ & $\mathrm{V}_{\mathrm{B}}$ & $-0.28<60$ \\
\hline $\mathrm{A}-\mathrm{B}$ & $\mathrm{I}_{\mathrm{AB}}$ & $\mathrm{V}_{\mathrm{AB}}$ & $\mathrm{V}_{\mathrm{CA}}$ & $-0.33<85$ \\
\hline
\end{tabular}




\begin{tabular}{|l|c|c|c|l|}
\hline $\mathrm{B}-\mathrm{C}$ & $\mathrm{I}_{\mathrm{BC}}$ & $\mathrm{V}_{\mathrm{BC}}$ & $\mathrm{V}_{\mathrm{AB}}$ & $-0.33<85$ \\
\hline $\mathrm{C}-\mathrm{A}$ & $\mathrm{I}_{\mathrm{CA}}$ & $\mathrm{V}_{\mathrm{CA}}$ & $\mathrm{V}_{\mathrm{BC}}$ & $-0.33<85$ \\
\hline
\end{tabular}

\section{Mho element No.4}

Operating equations:-

$$
\begin{aligned}
& \mathrm{S}_{1}=\mathrm{V}_{\Phi}-\mathrm{I}_{\Phi} \mathrm{Z}_{\mathrm{C}} \\
& \mathrm{S}_{2}=\mathrm{V}_{\mathrm{P}}
\end{aligned}
$$

\begin{tabular}{|c|c|c|c|}
\hline Element type & $\mathrm{I}_{\Phi}$ & $\mathbf{V}_{\Phi}$ & $\mathbf{V}_{\mathrm{P}}$ \\
\hline $\mathrm{A}-\mathrm{G}$ & $\mathrm{I}_{\mathrm{A}}+\mathrm{k}_{\mathrm{o}} \mathrm{I}_{\mathrm{N}}$ & $\mathrm{V}_{\mathrm{A}}$ & $0.2 \mathrm{~V}_{1 \mathrm{R}}(\mathrm{m})+0.8 \mathrm{~V}_{1 \mathrm{R}}$ \\
\hline $\mathrm{B}-\mathrm{G}$ & $\mathrm{I}_{\mathrm{B}}+\mathrm{k}_{\mathrm{o}} \mathrm{I}_{\mathrm{N}}$ & $\mathrm{V}_{\mathrm{B}}$ & $\left(0.2 \mathrm{~V}_{1 \mathrm{R}}(\mathrm{m})+0.8 \mathrm{~V}_{1 \mathrm{R}}\right) \mathrm{a}^{2}$ \\
\hline $\mathrm{C}-\mathrm{G}$ & $\mathrm{I}_{\mathrm{C}}+\mathrm{k}_{\mathrm{o}} \mathrm{I}_{\mathrm{N}}$ & $\mathrm{V}_{\mathrm{C}}$ & $\left(0.2 \mathrm{~V}_{1 \mathrm{R}}(\mathrm{m})+0.8 \mathrm{~V}_{1 \mathrm{R}}\right) \mathrm{a}$ \\
\hline $\mathrm{A}-\mathrm{B}$ & $\mathrm{I}_{\mathrm{AB}}$ & $\mathrm{V}_{\mathrm{AB}}$ & $\left(0.2 \mathrm{~V}_{1 \mathrm{R}}(\mathrm{m})+0.8 \mathrm{~V}_{1 \mathrm{R}}\right)\left(1-\mathrm{a}^{2}\right)$ \\
\hline $\mathrm{B}-\mathrm{C}$ & $\mathrm{I}_{\mathrm{BC}}$ & $\mathrm{V}_{\mathrm{BC}}$ & $\left(0.2 \mathrm{~V}_{1 \mathrm{R}}(\mathrm{m})+0.8 \mathrm{~V}_{1 \mathrm{R}}\right)\left(\mathrm{a}^{2}-\mathrm{a}\right)$ \\
\hline $\mathrm{C}-\mathrm{A}$ & $\mathrm{I}_{\mathrm{CA}}$ & $\mathrm{V}_{\mathrm{CA}}$ & $\left(0.2 \mathrm{~V}_{1 \mathrm{R}}(\mathrm{m})+0.8 \mathrm{~V}_{1 \mathrm{R}}\right)(\mathrm{a}-1)$ \\
\hline
\end{tabular}

Where $\mathrm{V}_{1 \mathrm{R}}=$ positive sequence voltage with respect to A-phase.

$$
\mathrm{V}_{1 \mathrm{R}}(\mathrm{m})=\text { memorized positive sequence voltage. }
$$

\section{Appendix (C)}

\section{Tables}

Table(1) system data for fig.(1)

\begin{tabular}{|c|c|c|c|c|c|}
\hline $\begin{array}{c}\text { Network } \\
\text { componen } \\
\mathrm{t}\end{array}$ & $\begin{array}{c}\text { MVA } \\
\text { Rating }\end{array}$ & $\begin{array}{c}\text { Voltage } \\
\text { rating } \\
\mathrm{kV}\end{array}$ & $\begin{array}{c}\mathrm{Z}_{1} \\
\Omega\end{array}$ & $\begin{array}{c}\mathrm{Z}_{2} \\
\Omega\end{array}$ & $\begin{array}{c}\mathrm{Z}_{\mathrm{o}} \\
\Omega\end{array}$ \\
\hline $\mathrm{G}_{1}$ & 200 & 15 & $0+\mathrm{j} 0.45$ & $0+\mathrm{j} 0.45$ & $0+\mathrm{j} 0.1125$ \\
\hline
\end{tabular}




\begin{tabular}{|c|c|c|c|c|c|}
\hline $\mathrm{G}_{2}$ & 200 & 15 & $0+\mathrm{j} 0.45$ & $0+\mathrm{j} 0.45$ & $0+\mathrm{j} 0.1125$ \\
\hline $\mathrm{T}_{1}$ & 200 & $15 / 115$ & $0+\mathrm{j} 26.45$ & $0+\mathrm{j} 26.45$ & $0+\mathrm{j} 26.45$ \\
\hline $\mathrm{T}_{2}$ & 200 & $15 / 115$ & $0+\mathrm{j} 26.45$ & $0+\mathrm{j} 26.45$ & $0+\mathrm{j} 26.45$ \\
\hline $\mathrm{TL}$ & 200 & 115 & $0.02+\mathrm{j} 79$. & $0.02+\mathrm{j} 79$. & $0.02+\mathrm{j} 26$. \\
& & & 35 & 35 & 45 \\
\hline
\end{tabular}

Table(2) apparent impedance for the types of mho relay When LG fault occurs .

\begin{tabular}{|c|c|c|c|c|}
\hline $\begin{array}{l}\mathrm{Rf} \\
(\Omega)\end{array}$ & & $\begin{array}{l}\text { Fault at } \mathrm{F}_{1} \\
\mathrm{Z}_{\mathrm{R}}=0.8 \mathrm{Z}_{\mathrm{L}}\end{array}$ & $\begin{array}{l}\text { Fault at } F_{2} \\
Z_{R}=0.8 Z_{L}\end{array}$ & $\begin{array}{l}\text { Fault at } F_{3} \\
Z_{R}=1.2 Z_{L}\end{array}$ \\
\hline \multirow[t]{4}{*}{0} & $\mathrm{Za}$ & $\begin{array}{l}0.01+\mathrm{j} 0.1 \\
7\end{array}$ & $-0.05+\mathrm{j} 0.3$ & $\begin{array}{l}0.02+\mathrm{j} 0.4 \\
3\end{array}$ \\
\hline & $\mathrm{Za}$ & $\begin{array}{l}0.03+\mathrm{j} 0.1 \\
7\end{array}$ & $-0.03+0.3$ & $\begin{array}{l}0.03+\mathrm{j} 0.4 \\
2\end{array}$ \\
\hline & $\begin{array}{l}\mathrm{Za} \\
3\end{array}$ & $\begin{array}{l}0.05+\mathrm{j} 0.2 \\
5\end{array}$ & $0+\mathrm{j} 0.4$ & $\begin{array}{l}0.09+\mathrm{j} 0.4 \\
9\end{array}$ \\
\hline & $\begin{array}{l}\mathrm{Za} \\
4\end{array}$ & $\begin{array}{l}0.01+\mathrm{j} 0.3 \\
3\end{array}$ & $0.01+0.05$ & $\begin{array}{l}0.02+\mathrm{j} 0.4 \\
8\end{array}$ \\
\hline \multirow[t]{4}{*}{5} & $\mathrm{Za}$ & $0.3+\mathrm{j} 0.19$ & $0.4+\mathrm{j} 0.4$ & $\begin{array}{l}0.36+\mathrm{j} 0.4 \\
6\end{array}$ \\
\hline & $\mathrm{Za}$ & $\begin{array}{l}0.31+\mathrm{j} 0.1 \\
9\end{array}$ & $0.4+\mathrm{j} 0.4$ & $\begin{array}{l}0.37+\mathrm{j} 0.4 \\
6\end{array}$ \\
\hline & $\mathrm{Za}$ & $\begin{array}{l}0.32+\mathrm{j} 0.2 \\
6\end{array}$ & $0.4+\mathrm{j} 0.5$ & $\begin{array}{l}0.37+\mathrm{j} 0.5 \\
2\end{array}$ \\
\hline & $\begin{array}{l}\mathrm{Za} \\
4\end{array}$ & $\begin{array}{l}0.17+\mathrm{j} 0.3 \\
2\end{array}$ & $0.3+\mathrm{j} 0.5$ & $\begin{array}{l}0.26+\mathrm{j} 0.4 \\
8\end{array}$ \\
\hline \multirow[t]{4}{*}{10} & $\mathrm{Za}$ & $\begin{array}{l}0.59+\mathrm{j} 0.2 \\
2\end{array}$ & $0.76+\mathrm{j} 0.47$ & $0.72+\mathrm{j} 0.5$ \\
\hline & $\mathrm{Za}$ & $\begin{array}{l}0.61+\mathrm{j} 0.2 \\
2\end{array}$ & $0.78+\mathrm{j} 0.5$ & $0.71+\mathrm{j} 0.5$ \\
\hline & $\mathrm{Za}$ & $\begin{array}{l}0.62+\mathrm{j} 0.2 \\
9\end{array}$ & $0.76+\mathrm{j} 0.56$ & $\begin{array}{l}0.71+\mathrm{j} 0.5 \\
7\end{array}$ \\
\hline & $\begin{array}{l}\mathrm{Za} \\
4\end{array}$ & $\begin{array}{l}0.34+\mathrm{j} 0.2 \\
7\end{array}$ & $0.51+\mathrm{j} 0.4$ & $\begin{array}{l}0.51+\mathrm{j} 0.3 \\
9\end{array}$ \\
\hline \multirow[t]{4}{*}{15} & $\mathrm{Za}$ & $0.9+\mathrm{j} 0.2$ & $1.15+\mathrm{j} 0.5$ & $\begin{array}{l}1.08+\mathrm{j} 0.5 \\
4\end{array}$ \\
\hline & $\begin{array}{l}\mathrm{Za} \\
2\end{array}$ & $\begin{array}{l}0.92+\mathrm{j} 0.2 \\
4\end{array}$ & $1.17+\mathrm{j} 0.52$ & $\begin{array}{l}1.08+\mathrm{j} 0.5 \\
4\end{array}$ \\
\hline & $\mathrm{Za}$ & $0.95+\mathrm{j} 0.3$ & $1.14+\mathrm{j} 0.65$ & $\begin{array}{l}1.08+j 0.6 \\
6\end{array}$ \\
\hline & $\mathrm{Za}$ & $0.5+\mathrm{j} 0.17$ & $0.7+\mathrm{j} 0.23$ & $\begin{array}{l}0.72+\mathrm{j} 0.2 \\
2\end{array}$ \\
\hline
\end{tabular}




\begin{tabular}{|l|l|l|l|l|}
\hline 20 & $\mathrm{Za}$ & $1.2+\mathrm{j} 0.26$ & $1.6+\mathrm{j} 0.51$ & $1.44+\mathrm{j} 0.6$ \\
& 1 & & & 2 \\
\cline { 2 - 5 } & $\mathrm{Za}$ & $1.2+\mathrm{j} 0.27$ & $1.6+\mathrm{j} 0.54$ & $1.37+\mathrm{j} 0.7$ \\
& 2 & & & 2 \\
\cline { 2 - 5 } & $\mathrm{Za}$ & $1.28+\mathrm{j} 0.4$ & $1.6+\mathrm{j} 0.77$ & $0.9+\mathrm{j} 0.64$ \\
3 & 2 & & \\
\cline { 2 - 5 } & $\mathrm{Za}$ & $0.61+\mathrm{j} 0.0$ & $0.82+\mathrm{j} 0.01$ & $-0.16-0.57$ \\
& 6 & 6 & & \\
\hline
\end{tabular}

Table(3) an apparent impedance for the types of mho relays When DLG fault occurs.

\begin{tabular}{|c|c|c|c|c|}
\hline $\mathrm{R}_{\mathrm{F}}$ & & $\begin{array}{l}\text { Fault at } F_{1} \\
Z_{R}=0.8 Z_{L}\end{array}$ & $\begin{array}{l}\text { Fault at } \mathrm{F}_{2} \\
\mathrm{Z}_{\mathrm{R}}=0.8 \mathrm{Z}_{\mathrm{L}}\end{array}$ & $\begin{array}{l}\text { Fault at } F_{3} \\
Z_{R}=1.2 Z_{L}\end{array}$ \\
\hline \multirow[t]{4}{*}{0} & $\begin{array}{l}\mathrm{Za} \\
1\end{array}$ & $0.0+\mathrm{j} 0.32$ & $-0.001+j 0.6$ & $\begin{array}{l}- \\
0.005+\mathrm{j} 0.6 \\
2\end{array}$ \\
\hline & $\begin{array}{l}\mathrm{Za} \\
2\end{array}$ & $0.0+\mathrm{j} 0.32$ & $0.005+$ jo. 6 & $\begin{array}{l}0.001+\mathrm{j} 0.6 \\
2\end{array}$ \\
\hline & $\begin{array}{l}\mathrm{Za} \\
3\end{array}$ & $\begin{array}{l}- \\
0.05+\mathrm{j} 0.3 \\
1\end{array}$ & $-0.05+j 0.64$ & $-0.05+j 0.63$ \\
\hline & $\begin{array}{l}\mathrm{Za} \\
4\end{array}$ & $\begin{array}{l}- \\
0.02+\mathrm{j} 0.2 \\
9\end{array}$ & $-0.06+\mathrm{j} 0.49$ & $-0.05+\mathrm{j} 0.43$ \\
\hline
\end{tabular}




\begin{tabular}{|c|c|c|c|c|}
\hline \multirow[t]{4}{*}{5} & $\begin{array}{l}\mathrm{Za} \\
1\end{array}$ & $\begin{array}{l}0.13+\mathrm{j} 0.3 \\
2\end{array}$ & $0.16+j 0.64$ & $0.16+\mathrm{j} 0.64$ \\
\hline & $\begin{array}{l}\mathrm{Za} \\
2\end{array}$ & $\begin{array}{l}0.14+\mathrm{j} 0.3 \\
2\end{array}$ & $0.17+\mathrm{j} 0.64$ & $0.16+\mathrm{j} 0.64$ \\
\hline & $\begin{array}{l}\mathrm{Za} \\
3\end{array}$ & $\begin{array}{l}0.08+\mathrm{j} 0.3 \\
2\end{array}$ & $0.12+\mathrm{j} 0.64$ & $0.11+\mathrm{j} 0.64$ \\
\hline & $\begin{array}{l}\mathrm{Za} \\
4\end{array}$ & $0.04+\mathrm{j} 0.3$ & $0.06+\mathrm{j} 0.52$ & $0.05+\mathrm{j} 0.47$ \\
\hline \multirow[t]{4}{*}{10} & $\begin{array}{l}\mathrm{Za} \\
1\end{array}$ & $\begin{array}{l}0.24+\mathrm{j} 0.3 \\
3\end{array}$ & $0.32+\mathrm{j} 0.65$ & $0.31+\mathrm{j} 0.66$ \\
\hline & $\begin{array}{l}\mathrm{Za} \\
2\end{array}$ & $\begin{array}{l}0.25+\mathrm{j} 0.3 \\
3\end{array}$ & $0.33+\mathrm{j} 0.66$ & $0.32+\mathrm{j} 0.67$ \\
\hline & $\begin{array}{l}\mathrm{Za} \\
3\end{array}$ & $\begin{array}{l}0.19+\mathrm{j} 0.3 \\
4\end{array}$ & $0.28+\mathrm{j} 0.64$ & $0.27+\mathrm{j} 0.66$ \\
\hline & $\begin{array}{l}\mathrm{Za} \\
4\end{array}$ & $0.09+\mathrm{j} 0.3$ & $0.21+\mathrm{j} 0.5$ & $0.2+\mathrm{j} 0.47$ \\
\hline \multirow[t]{4}{*}{15} & $\begin{array}{l}\mathrm{Za} \\
1\end{array}$ & $\begin{array}{l}0.38+\mathrm{j} 0.3 \\
3\end{array}$ & $0.5+\mathrm{j} 0.67$ & $0.47+\mathrm{j} 0.69$ \\
\hline & $\begin{array}{l}\mathrm{Za} \\
2\end{array}$ & $\begin{array}{l}0.39+\mathrm{j} 0.3 \\
3\end{array}$ & $0.5+\mathrm{j} 0.67$ & $0.47+\mathrm{j} 0.69$ \\
\hline & $\begin{array}{l}\mathrm{Za} \\
3\end{array}$ & $\begin{array}{l}0.31+\mathrm{j} 0.3 \\
4\end{array}$ & $0.44+\mathrm{j} 0.63$ & $0.42+\mathrm{j} 0.66$ \\
\hline & $\begin{array}{l}\mathrm{Za} \\
4\end{array}$ & $\begin{array}{l}0.15+\mathrm{j} 0.2 \\
7\end{array}$ & $0.34+\mathrm{j} 0.42$ & $0.33+\mathrm{j} 0.39$ \\
\hline \multirow[t]{2}{*}{20} & $\begin{array}{l}\mathrm{Za} \\
1\end{array}$ & $0.5+\mathrm{j} 0.34$ & $0.67+\mathrm{j} 0.67$ & $0.65+\mathrm{j} 0.7$ \\
\hline & $\begin{array}{l}\mathrm{Za} \\
2\end{array}$ & $\begin{array}{l}0.52+\mathrm{j} 0.3 \\
5\end{array}$ & $0.68+\mathrm{j} 0.68$ & $0.65+\mathrm{j} 0.71$ \\
\hline
\end{tabular}




\begin{tabular}{|l|l|l|l|l|}
\hline & $\mathrm{Za}$ & $0.43+\mathrm{j} 0.3$ & $0.6+\mathrm{j} 0.62$ & $0.58+\mathrm{j} 0.64$ \\
3 & 4 & & \\
\cline { 2 - 5 } & $\mathrm{Za}$ & $0.2+\mathrm{j} 0.24$ & $0.42+\mathrm{j} 0.29$ & $0.43+\mathrm{j} 0.25$ \\
4 & & & \\
\hline
\end{tabular}

Appendix (D)

Figures

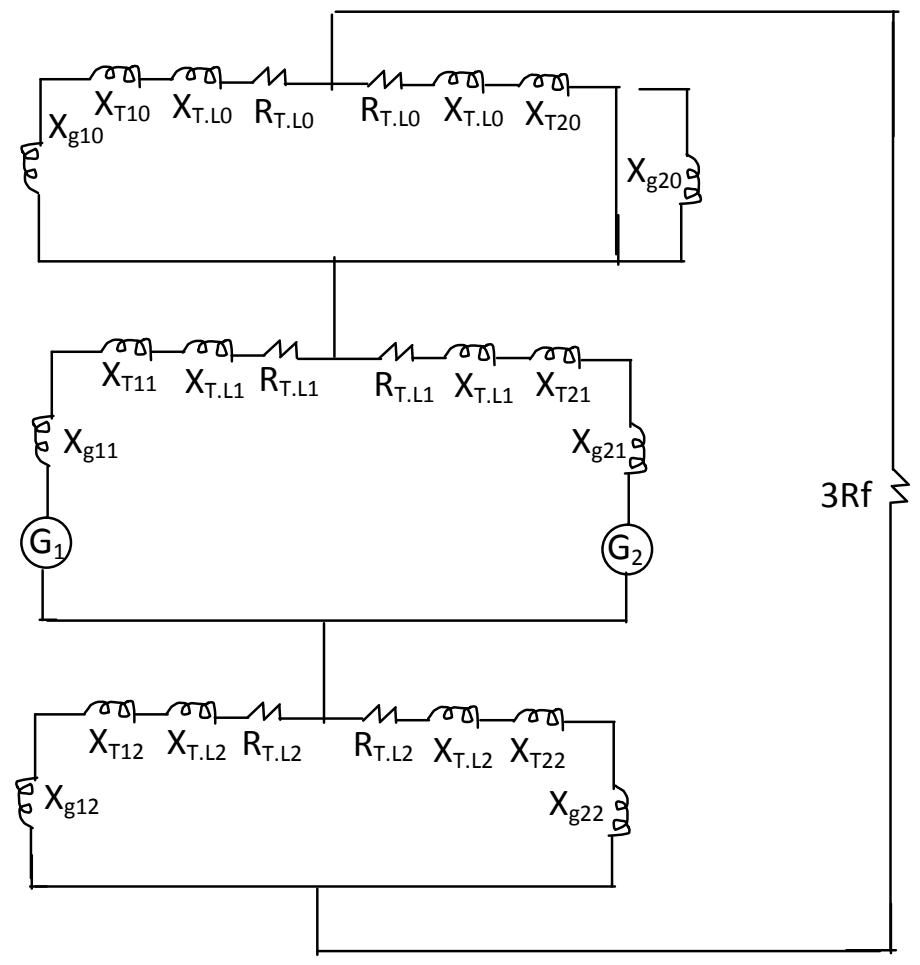

Fig(2) equivalent circuit of network for (LG) fault 


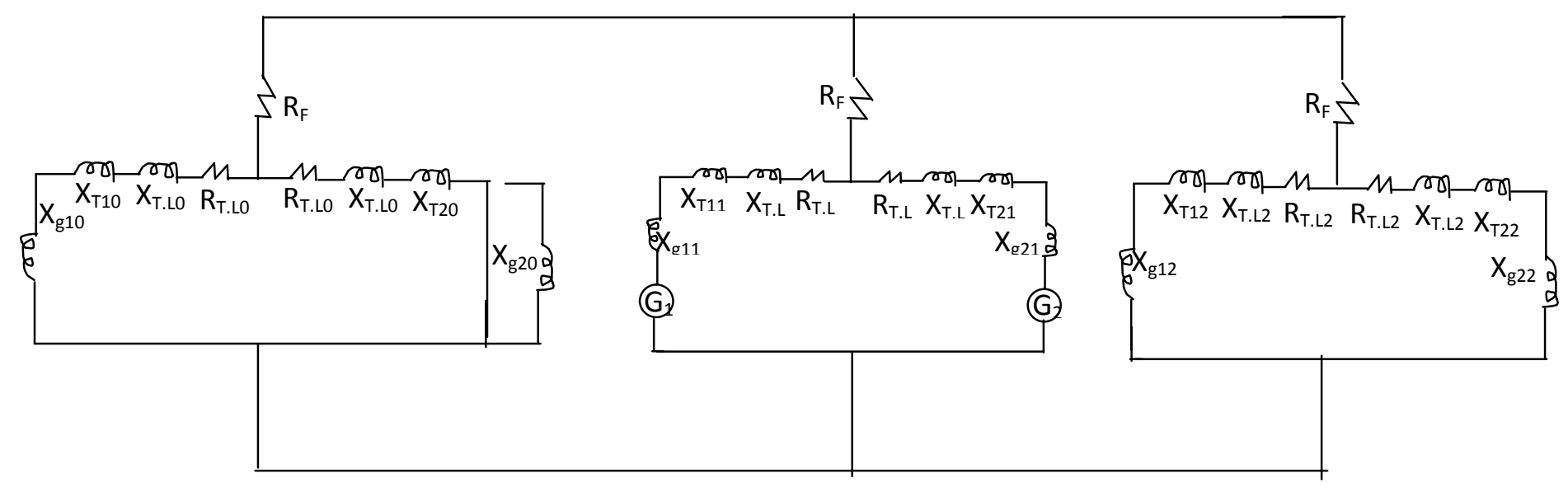

Fig(3) equivalent circuit of network for (DLG) fault

The work was carried out at the college of Engg. University of Mosul 